\title{
Diagnosis of cyclic Cushing syndrome using the morning urine free cortisol to creatinine ratio
}

Yi Sun Jang, Ihn Suk Lee, Jong Min Lee, Soo A Choi, Gi Jun Kim, and Hye Soo Kim

Department of Internal Medicine, College of Medicine, The Catholic University of Korea, Seoul, Korea

Received: October 16, 2014

Revised : December 1, 2014

Accepted: December 15, 2014

\section{Correspondence to}

Hye Soo Kim, M.D.

Department of Internal Medicine, College of Medicine, Daejeon St. Mary's Hospital, The Catholic University of Korea, 64 Daeheung-ro, Jung-gu, Daejeon 34943, Korea Tel: +82-42-220-9114

Fax: +82-42-252-6807

E-mail:drkhs@catholic.ac.kr
To the Editor,

Cortisol fluctuations in Cushing syndrome were first detected in 1956 and named cyclic Cushing syndrome [1]. Cycle lengths have been reported, varying between 12 hours and 85 days with at least three peaks and two troughs [2]. To assess cortisol production cycles, repeated hormone tests are necessary. According to the literature $[2,3]$, tests to measure the early morning first-voided urine free cortisol to creatinine ratio (UFCCR) or salivary free cortisol can be used as feasible alternatives to assess cyclic cortisol overproduction because they are more convenient to perform. Here, we report a case of cyclic Cushing syndrome that was diagnosed using consecutive UFCCR, reported for the first time in Korea.

An 18-year-old girl was admitted to the gastroenterology department with the chief complaints of nausea and weakness and was referred to an endocrinologist owing to high blood glucose and obesity in November 2009. Her body mass index was $31.24 \mathrm{~kg} / \mathrm{m}^{2}$ (height $162 \mathrm{~cm}$, weight $82 \mathrm{~kg}$ ). Her blood pressure was 100/60 mmHg. She was obese, and her face was round (Fig. $1 \mathrm{~A}$ ), but she did not show other cushingoid features such as a buffalo hump or abdominal striae. On physical examination, hepatomegaly and mild tenderness on the right upper abdomen were found. She denied progressive weight gain. She had been consuming herbal medications and oral contraceptive pills during the previous 2 months owing to irregular menstrual cycles. She complained of episodic symptoms such as plethora, feelings of edema, and lethargy. She reported that the durations of episodic symptoms were approximately 5 to 7 days with or without menstruation. Her father was on renal replacement therapy for diabetic end-stage renal disease, and he was consuming medications for panhypopituitarism secondary to pituitary hemorrhage, which had occurred during hemorrhagic fever with renal failure. Her mother had a past medical history of chronic myeloid leukemia. Based on a fasting blood glucose level of $297 \mathrm{mg} / \mathrm{dL}$ and glycated hemoglobin $(\mathrm{HbAlc})$ of $8.6 \%$, diabetes mellitus was diagnosed for the first time. The fasting C-peptide level was $3.0 \mathrm{ng} / \mathrm{mL}$, and autoantibodies, such as anti-glutamic acid decarboxylase antibody and islet cell antibody, were all negative. Total bilirubin was normal. Aspartate aminotransferase (AST) and alanine aminotransferase (ALT) levels were elevated to 397 and $190 \mathrm{IU} / \mathrm{L}$, respectively. Viral markers and autoantibodies for hepatitis were not detected. Total cholesterol, triglycerides, and high density lipoprotein cholesterol (HDL-C) levels were 330, 1,012, and $16.9 \mathrm{mg} / \mathrm{dL}$, respectively. Her initial adrenocorticotropic hormone (ACTH) and cortisol levels 

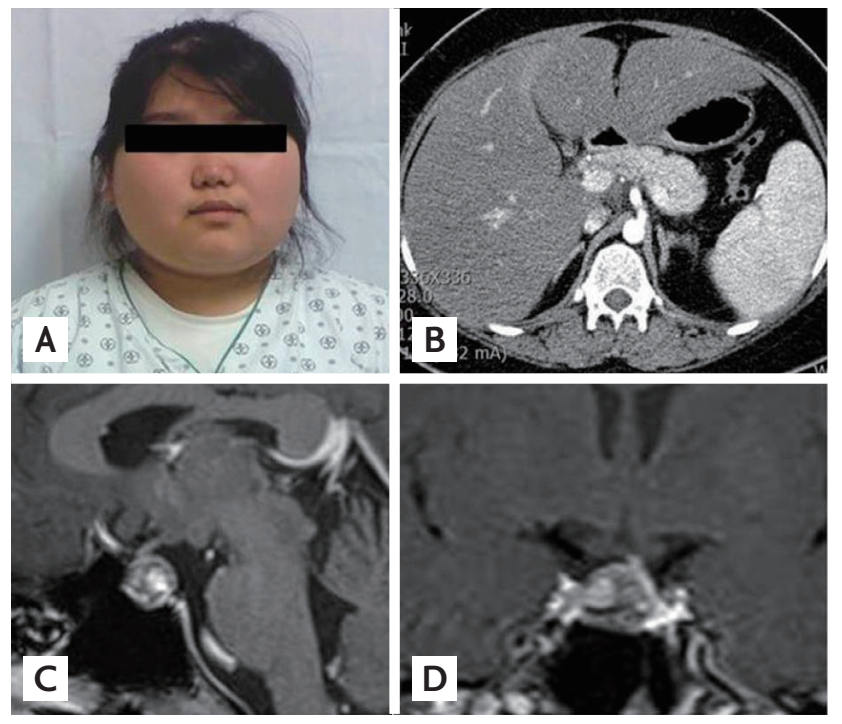

Figure 1. (A) Round and plethoric face of the patient. (B) Abdomen computed tomography of the patient showing normal adrenal glands. (C, D) Sellar magnetic resonance imaging of the patient showing a $1.5-\mathrm{cm}$, markedly heterogeneous pituitary macroadenoma with left-sided stalk deviation.

were $17.81 \mathrm{pg} / \mathrm{dL}$ and $15.5 \mu \mathrm{g} / \mathrm{dL}$, respectively. The result of the thyroid function test was normal. The prolactin level was $13.15 \mathrm{ng} / \mathrm{mL}$; levels of luteinizing hormone, follicle stimulating hormone, and estradiol were 5.26, 6.79, and $5.00 \mathrm{IU} / \mathrm{L}$, respectively. Growth hormone and insulin-like growth factor-1 levels were 0.46 and $40.2 \mathrm{ng} / \mathrm{mL}$, respectively. Flat abdomen film and abdomen computed tomography (Fig. 1B) showed marked hepatosplenomegaly and diffuse gallbladder wall edema with normal adrenal glands. Her liver function and hepatomegaly slowly improved with conservative therapy. A total of 56 units of insulin daily were needed for glucose control at that time, and statin and fenofibrate were prescribed.

An overnight dexamethasone suppression test (DST) revealed that the cortisol level at 8:00 AM was 17.3 $\mu \mathrm{g} /$ dL. During low-dose DST, serum cortisol and 24-hour urine free cortisol (UFC) were not sufficiently suppressed (serum cortisol $6.63 \mu \mathrm{g} / \mathrm{dL}$, UFC $17.5 \mu \mathrm{g} /$ day), and cortisol was paradoxically elevated after high-dose DST. The baseline cortisol level was $15.14 \mu \mathrm{g} / \mathrm{dL}$, and, after highdose DST, the cortisol level increased to $63.44 \mu \mathrm{g} / \mathrm{dL}$. The basal plasma ACTH level was $23.33 \mathrm{pg} / \mathrm{mL}$, and the ACTH level after high-dose DST was $86.02 \mathrm{pg} / \mathrm{mL}$. The baseline 24-hour UFC was $28.8 \mu \mathrm{g} /$ day (normal reference range, 20 to 90) and strikingly increased to $4,387 \mu \mathrm{g} /$ day on the last day of the high-dose DST. During high-dose DST, she complained of headache, plethora, lethargy, edema, and an unpleasant feeling similar to her previous episodic symptoms. Sellar magnetic resonance imaging showed a 1.5-cm heterogeneous pituitary macroadenoma, indicating recent hemorrhage (Fig. $1 \mathrm{C}$ and $\mathrm{1D}$ ). We recommended an operation to treat this atypical pituitary Cushing syndrome. However, she wanted to be referred to Severance Hospital for treatment of the pituitary tumor. The doctors at that hospital decided to observe the patient instead of immediate surgery after repeating the lowand high-dose DST. They discontinued all medications during the observation period as her clinical condition had improved, and she remained well without any medications or insulin for a period of time. The results of her blood test at our outpatient department in March 2010 were as follows: ACTH, 56.95 pg/mL; cortisol, $13.41 \mu \mathrm{g} / \mathrm{dL}$; HbAıc, 6.1\%; AST, 54 IU/L; ALT, 88 IU/L; total cholesterol, $253 \mathrm{mg} / \mathrm{dL}$; triglycerides, $214 \mathrm{mg} / \mathrm{dL}$; HDL-C, $32.1 \mathrm{mg} / \mathrm{dL}$; and low density lipoprotein cholesterol $193.4 \mathrm{mg} / \mathrm{dL}$. We randomly performed serial early morning UFCCR for 7 days under the suspicion of cyclic Cushing syndrome and detected a 7 -fold increase in UFCCR from her usual UFCCR (Fig. 2). One month later, a 30-day sequential early morning UFCCR was performed, and the results showed that the cortisol peak between days 22 and 26 was 200fold higher than that of the other days (Fig. 2). She also described episodic symptoms, as she had experienced

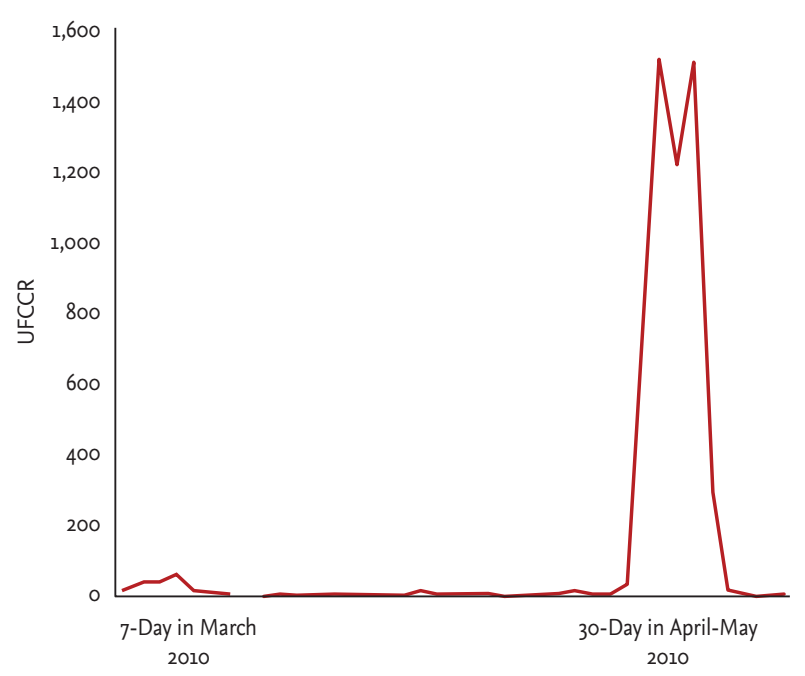

Figure 2. Sequential morning urine free cortisol to creatinine ratio (UFCCR) in the patient for 7 days in March and 30 days from April to May 2010. 
previously, during the peak UFCCR period. It appeared that the cortisol levels cyclically increased approximately once a month. In November 2010, she underwent a pituitary operation via the transsphenoidal approach at Severance Hospital. Histopathology revealed that the tumor cells were arranged in a trabecular and diffuse pattern (Fig. 3A), with granular eosinophilic cytoplasm and occasionally forming focal pseudovascular rosettes (Fig. 3B). Immunohistochemistry for pituitary hormones was positive only for ACTH (Fig. $3 \mathrm{C}$ and $3 \mathrm{D}$ ). The postoperative combined pituitary test showed panhypopituitarism. After the operation, hormone replacement therapy was commenced with corticosteroids and L-thyroxine. She did not experience any further episodic symptoms, and her diabetes was controlled well with metformin. She remained amenorrheic after the operation, and was prescribed cyclic hormone replacement therapy. She lost approximately $10 \mathrm{~kg}$ over a period of 3 years postoperatively.

The prevalence of cyclic Cushing syndrome is unknown, but that of cyclic hypercortisolism is estimated at approximately $20 \%$ to $40 \%$. ACTH-dependent pituitary adenoma is the most frequent cause of cyclic Cushing syndrome [4]. To diagnose cyclic Cushing syndrome, it is important to confirm cyclic cortisol secretion. However, existing screening tests for Cushing syndrome, such as the 24-hour UFC and 1-mg overnight DST, are not ideal to assess cyclic hypercortisolism. Based on the need for a more convenient, repeatable test, early morning first-voided UFCCR has been used to diagnose cyclic Cushing syndrome $[2,3]$. This test reflects a mixture of diurnal nadir to the peak secretion of cortisol because it uses urine collected in the bladder from midnight to early morning. The sensitivity and specificity of spot urine cortisol to creatinine ratio are unknown. Only Atkinson et al, with 46 samples, reported a positive correlation between early morning UFCCR and 24-hour UFC levels $(r=0.92)$ and determined that the upper range of the normal cortisol to creatinine ratio was 50 [3].

The excellent repeatability is a major advantage of the UFCCR. If the patient has to urinate frequently during the night, the first-voided UFCCR level will not accurately reflect the serum cortisol level. Therefore, clear instructions to refrain from urinating during the night should be provided. According to a previous study, 9.33\% of patients with simple obesity were diagnosed with Cushing syndrome [5]. Therefore, if there is a clinical sus-

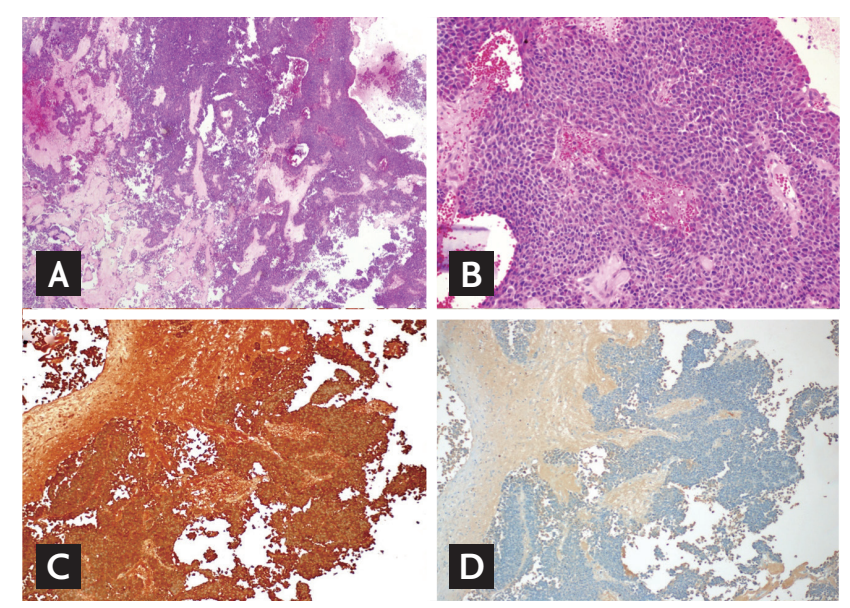

Figure 3. Tissue pathology of the pituitary tumor. The tumor cells were arranged in a trabecular and diffuse pattern (A: $\mathrm{H} \& \mathrm{E}, \times 40)$. They were with granular eosinophilic cytoplasm, occasionally forming focal pseudovascular rosettes (B: H\&E, $\times 200$ ). On immunohistochemistry, the tumor was positive for adrenocorticotropic hormone (C: ACTH, ×100), but growth hormone (D: GH, x100), prolactin, thyroid stimulating hormone, follicle stimulating hormone, and luteinizing hormone were all negative (data not shown). These pictures were provided by courtesy of Dr. Se Hoon Kim in Yonsei University.

picion of Cushing syndrome, even due to simple obesity, screening for excess cortisol production should be performed, and tests for cyclical production of cortisol may also be performed despite normal screening test results for Cushing syndrome. For this reason, a simple and repeatable screening test for Cushing syndrome is necessary, and early morning UFCCR could be an alternative tool for this purpose.

Keywords: Cyclic Cushing syndrome; Morning urine free cortisol to creatinine ratio

\section{Conflict of interest}

No potential conflict of interest relevant to this article was reported.

\section{REFERENCES}

1. Birke G, Diczfalusy E. Fluctuation in the excretion of adrenocortical steroids in a case of Cushing's syndrome. J Clin Endocrinol Metab 1956;16:286-290.

2. Mullan KR, Atkinson AB, Sheridan B. Cyclical Cushing's syndrome: an update. Curr Opin Endocrinol Dia- 
betes Obes 2007;14:317-322.

3. Atkinson AB, Kennedy AL, Carson DJ, Hadden DR, Weaver JA, Sheridan B. Five cases of cyclical Cushing's syndrome. Br Med J (Clin Res Ed) 1985;291:1453-1457.

4. Meinardi JR, Wolffenbuttel BH, Dullaart RP. Cyclic
Cushing's syndrome: a clinical challenge. Eur J Endocrinol 2007;157:245-254.

5. Tiryakioglu O, Ugurlu S, Yalin S, et al. Screening for Cushing's syndrome in obese patients. Clinics (Sao Paulo) 2010;65:9-13. 